\title{
A case report on management of cervical insufficiency using Chhattisgarh balloon in second trimester cerclage for fetal membrane prolapse
}

\author{
Shefali Tyagi, Swapnil Bhagat, Shreeja Karan \\ Correspondence: Dr. Shefali Tyagi; Senior Consultant, Department of Obstetrics and \\ Gynecology, Cloudnine Hospital, Karnataka, India; Email - shefali.tyagi@hotmail.com \\ Distributed under Attribution-Non Commercial-Share Alike 4.0 International (CC BY-NC-SA 4.0)
}

\begin{abstract}
Many second trimester miscarriages and neonatal deaths are caused by cervical incompetence. In second trimester if the membranes prolapse in vagina due to cervical incompetence it can be technically very difficult for the clinician to push the membranes back in cervix and apply cerclage to prolong the pregnancy. We present a case of 30 year old primi gravida who came to emergency at 22 weeks of gestation with cervix dilated to $4 \mathrm{cms}$ and membranes prolapsing till the vagina. After ruling out possibility of infection, decision for rescue cerclage was taken. The membranes were deposited back in the uterus with help of Chhattisgarh balloon and Mac Donald's stitch was applied. Patient could successfully carry her pregnancy till 35 weeks and baby did not need NICU admission. Chhattisgarh balloon can be a useful tool in low resource country like India where it can be easily prepared, inexpensive and less traumatic compared to other alternatives available as of now.
\end{abstract}

Keywords: Cervical incompetence, membrane prolapse, Chhattisgarh balloon, MacDonald's stitch.

Many second trimester miscarriages and neonatal deaths are caused by cervical incompetence. In second trimester if the membranes prolapse in vagina due to cervical incompetence it can be technically very difficult for the clinician to push the membranes back in cervix and apply cerclage to prolong the pregnancy. Emergency cervical cerclage can play a major role inimproving the neonatal outcomes ${ }^{1}$.Chhattisgarh balloon was used to push membranes back into cervix followed by applying the MacDonald's Cerclage.

\section{Case report}

A 30 year old 22 weeks primigravida underwent usual anomaly scan where cervical opening ( cervix opened and membranes prolapsing out) was reported and she was referred by her primary gynaecologist to our hospital. Patient presented in our hospital in emergency with the ultrasound report. On examination her vitals were found to be stable. Speculum examination showed bulging membranes in vagina and it was also noted that there was no rupture of membranes or leaking of amniotic fluid vaginally. A vaginal swab for culture and sensitivity was taken and a gentle bimanual vaginal (PV) examination was done where it was found that the Os was $4 \mathrm{~cm}$ dilated and membranes

Received: $28^{\text {th }}$ August 2019. Accepted: $11^{\text {th }}$ March 2019.

Tyagi S, Bhagat S, Karan S. A case report on management of cervical insufficiency using Chhattisgarh balloon in second trimester cerclage for fetal membrane prolapse. The New Indian Journal of OBGYN. 2019; 6(1):60-2. 
were bulging till Os. In parallel patient's complete blood count $(\mathrm{CBC})$ and $\mathrm{C}$ reactive protein (CRP) were sent for examination, lab report showed total count was 10,040/ cumm and CRP was normal. Urine was sent for microscopy and culture. After explaining to the patient about the possibility of preterm labour, rupture of membranes and poor outcome of pregnancy, decision for cervical cerclage was taken with patient's consent. Patient was put on Inj Cefuroxime $1.5 \mathrm{gm} \mathrm{i} / \mathrm{v} \mathrm{BD}$, Inj Proluton depot $500 \mathrm{mg}$ intramuscular prior to cerclage procedure. Uterine tocolysis was given. To push the membranes back in uterus we used Chhattisgarh balloon, preparation steps as given by Nalini Mishra et al. ${ }^{2}$

Chhattisgarh balloon was prepared manually as follows -

1) Collected a Foley's catheter of size 20-22, a packed condom, scissors, two $20 \mathrm{ml}$ syringes and $500 \mathrm{ml}$ bottle of saline in a tray.

2) From the drainage tube of catheter, cut two rings of approximately 1-2 $\mathrm{mm}$ width.

3) Excised (not merely incise) the bulb of the catheter after inflating it with air.

4) Unfolded the condom over distal one third of the catheter.

5) Used these rings encircling twice only (like a rubber band in a ponytail) to secure the condom over catheter leaving $1.5-2 \mathrm{~cm}$ from both the ends of condom.

6) Excised tip of the Foley's catheter and condom which were washed with antiseptic solution.

The procedure was performed under general anaesthesia with the patient in lithotomy position. Urinary bladder was catheterized and emptied. Sims speculum was placed in the posterior vaginal wall first and then another narrow speculum was placed on the anterior vaginal wall. The anterior lip of cervix was held by sponge holder.

The balloon was prepared and inflated with $50 \mathrm{ml}$ of saline. The inflated condom was pushed inside the cervix to push the membranes inside the cervix and further inflated with around $100 \mathrm{ml}$ saline.

The Chhattisgarh balloon pushed the membranes up due to which the cervical margins were free to apply the Macdonald's cerclage. Mersilene tape (5mm) was used for the cerclage. The condom was deflated gradually and then taken out completely before tightening the cervical suture. The ultrasound was done in parallel to the procedure and showed membranes intact, post stitch distal cervix approximated to $1.5 \mathrm{cms}$.

The patient was continued on Cephalosporins (Cefuroxime) ( 5 days ) and Tocolysis ( 15 days). Micronized Progesterone (300 mg daily x 15 days) was also added.

Patient was discharged on third post-operative day. She followed up regularly in OPD where the stitch was checked and found to be in position. Rest of her pregnancy was uneventful. She went into labour at 35 weeks and after removal of the stitches, she delivered a female baby weighing $2.7 \mathrm{~kg}$ vaginally. Baby did not require NICU admission.

\section{Discussion}

Cervical cerclage was first proposed by Shirodkar in 1955 and then his technique was simplified by McDonald's in 1957. Treatment options with advanced cervical dilatation are limited to either expectant management or placement of an emergency cervical cerclage. During cerclage the prolapsed membranes must be placed back in the uterine cavity before the procedure to avoid high risk of iatrogenic PPROM. This is accomplished least traumatically by placing the patient in the lithotomy position with a steep Trendelenburg tilt, combined with the administration of tocolytics ${ }^{3}$. Urinary catheterization can also help to reposition the membranes in the uterus. However, a full bladder tends to reduce exposure of the operative field and push the cervix higher up into the pelvis. Invasive methods for reducing the fetal membranes include directly pushing them back with a smooth surfaced device, such as a Foley's catheter balloon, or pushing with gauze mounted on a clamp or an inflated balloon of the type used for endoscopic preperitoneal dissection, or a metreurynteror minimetreu (a rubber balloon device) inside the cervical os.

Alternatively, transabdominal amniocentesis and amnioreduction under ultrasound guidance can be performed ${ }^{4}$ in pregnant women with advanced bulging membranes. The patient was placed in the knee-chest position and a metreurynter with a bulb was inserted 
The New Indian Journal of OBGYN. 2019 (July-December); 6(1)

and inflated with warm saline solution. After the Protruding Membranes. International Scholarly Research membranes were pushed inside the uterine cavity Network. 2012; 2012(3): Article ID 842841

modified McDonald's suture was placed in a case of 24 weeks twin gestation reported by Tsapanos et al. ${ }^{5}$ where the prolongation of pregnancy was 14 weeks and the gestational age at the time of delivery 38 weeks was noted.

\section{Conclusion}

Cervical cerclage should be considered in patients with advanced cervical dilatation and bulging membranes in the second trimester. Despite overall poor prognosis in such cases successful outcomes may be obtained in selected cases. The Chhattisgarh balloon is a device which is easy to make, easy to use, cost effective and can be used in cases of secondtri mester prolapse of membranes to salvage the pregnancy.

\section{Conflict of interest: None. Disclaimer: Nil.}

\section{References}

1. Deb P, Aftab N, Muzaffar S. Prediction of Outcomes for Emergency Cervical Cerclage in the presence of

2. Mishra N, Agrawal S, Gulabani K, Shrivastava C. Use of an Innovative Condom Balloon Tamponade in Postpartum Haemorrhage: A Repor. Obstet Gynaecol India. 2016; 66(1): 63-7.

3. Prasad N, Thampan AS, Nagarathnamma R. Emergency cervical cerclage and pregnancy outcomes. Int J Reprod Contracept Obstet Gynecol. 2017; 6: 1993-8

4. Ogawa M, Sanada H, Tsuda A, Hirano H, Tanaka T. Modified cervical cerclage in pregnant women with advanced bulging membranes: Knee chest positioning. Acta Obstet Gynecol Scand. 1999; 78(9): 779-82.

5. Tsapanos VS, Decavalas GO, Adonakis GL, Kourounis GS. Late or emergency (salvage) cerclage of a dilated cervix after tissue support with pelvicol implant: A case. J Biomed Mater Res B Appl Biomater. 2005; 72(2): 368-72.

Shefali Tyagi ${ }^{1}$, Swapnil Bhagat ${ }^{2}$, Shreeja Karan ${ }^{3}$

${ }^{1}$ Consultant, Obstetrics and Gynaecology; ${ }^{2}$ Consultant Radiologist; ${ }^{3}$ Associate OBG, Cloud Nine Hospital, Bangalore, Karnataka, India. 